\title{
Assessment of Transport in NCSX
}

D. R. Mikkelsen ${ }^{1}$, H. Maassberg ${ }^{2}$, M. C. Zarnstorff ${ }^{1}$, C. D. Beidler ${ }^{2}$, W. A.

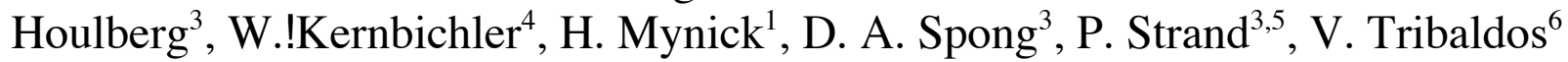

${ }^{1}$ Princeton Plasma Physics Laboratory

${ }^{2}$ Max Planck Institut für Plasmaphysik

${ }^{3}$ Oak Ridge National Laboratory

${ }^{4}$ Institut für Theoretische Physik, Technische Universität Graz

${ }^{5}$ Chalmers University of Technology

${ }^{6}$ Laboratorio Nacional de Fusión, CIEMAT

Proofs to:

D.R. Mikkelsen

Princeton Plasma Physics Laboratory

P.O. Box $451 \mathrm{MS}-34$

Princeton, New Jersey 08543

U.S.A.

dmikkelsen@pppl.gov

(609) 243-2624

Fax (609) 243-2874

Page charge invoice to:

Pamela Hampton

Princeton Plasma Physics Laboratory

P.O. Box 451 MS-38

Princeton, New Jersey 08543

U.S.A.

phampton@pppl.gov

(609) 243-2653

Fax (609) 243-2751

42 pages, no tables, 6 figures. 


\title{
Assessment of Transport in NCSX
}

\author{
D. R. Mikkelsen ${ }^{1}$, H. Maassberg ${ }^{2}$, M. C. Zarnstorff ${ }^{1}$, C. D. Beidler ${ }^{2}$, W. A. Houlberg ${ }^{3}$, \\ W. Kernbichler ${ }^{4}$, H. Mynick ${ }^{1}$, D. A. Spong ${ }^{3}$, P. Strand ${ }^{3,5}$, V. Tribaldos ${ }^{6}$ \\ ${ }^{1}$ Princeton Plasma Physics Laboratory \\ ${ }^{2}$ Max Planck Institut für Plasmaphysik \\ ${ }^{3}$ Oak Ridge National Laboratory \\ ${ }^{4}$ Institut für Theoretische Physik, Technische Universität Graz \\ ${ }^{5}$ Chalmers University of Technology \\ ${ }^{6}$ Laboratorio Nacional de Fusión, CIEMAT
}

Abstract

We explore whether the energy confinement and planned heating in NCSX are sufficient to test MHD stability limits, and whether the configuration is sufficiently quasi-axisymmetric to reduce the neoclassical ripple transport to low levels, thereby allowing tokamak-like transport. A 0-D model with fixed profile shapes is related to global energy confinement scalings for stellarators and tokamaks; neoclassical transport properties are assessed with the DKES, NEO, and NCLASS codes; and a power balance code is used to predict temperature profiles. Reaching the NCSX goal of $<$ beta $>=4 \%$ at low collisionality will require H_ISS-95=3, which is higher than the best achieved in present stellarators. However, this level of confinement is actually $\sim 10 \%$ lower than that predicted by the ITER-97P tokamak L-mode scaling. By operating near the stellarator density limit, the required H_ISS-95 is reduced by 35\%. The high degree of quasiaxisymmetry of the configuration and the self-consistent 'ambipolar' electric field reduce the neoclassical ripple transport to a small fraction of the neoclassical axisymmetric transport. A combination of neoclassical and anomalous transport models produces pressure profile shapes that are within the range of those used to study the MHD stability of NCSX. We find that $<$ beta $>=4 \%$ plasmas are 'neoclassically accessible', and are compatible with large levels of anomalous transport in the plasma periphery. 


\section{Introduction}

There are three major transport issues that impact the NCSX design [1]. First, is the energy confinement (with the available heating power) sufficient to reach the MHD stability limit? Second, is the configuration sufficiently quasi-axisymmetric to reduce the neoclassical ripple transport to low levels, thereby allowing tokamak-like transport? Third, is the projected pressure profile stable for $<\square>\sim 4 \%$ ?

The energy confinement needed to achieve the $<\square>=4 \%$ design goal is assessed chiefly through the required enhancement factor, H, relative to a standard stellarator scaling, ISS-95 [2], and the latest tokamak L-mode scaling, ITER-97P [3]. Comparison with a tokamak scaling is also warranted because there is a profoundly close relationship between the orbit drifts in a quasi-axisymmetric configuration and an equivalent axisymmetric configuration, and orbit drifts are at the heart of both neoclassical and microturbulent transport. The high degree of quasiaxisymmetry in NCSX effectively eliminates neoclassical helical ripple transport, reducing the neoclassical energy transport to essentially axisymmetric levels. Lowering the helical ripple also reduces the plasma viscosity [4]. When the parallel flow velocity deviates from its ambipolar value, the resulting non-ambipolar transport produces a radial current that exerts an opposing torque and viscously damps the flow deviation. The non-ambipolar radial transport arises entirely from the helical ripple transport, so the low effective ripple of NCSX leads directly to low viscosity. The reduction in viscosity may allow the partial suppression of microturbulence by permitting the development of turbulently driven zonal flows, as predicted in tokamaks. As a result, the ITER-97P L-mode scaling may be an appropriate predictor of the 'low-mode' 
confinement in NCSX. The experimental basis for these scalings is briefly reviewed in Section 1.1. Neoclassical transport theory for stellarators is compared with experiment in Section 1.2 because neoclassical transport plays a significant role in the power balance calculations described in Section 3. Enhanced confinement, i.e., above the ISS-95 scaling, is seen in many stellarators, and is discussed in Section 1.3. Confinement may also be enhanced in NCSX because the relatively low viscosity could permit large-scale sheared flows driven by neutral beam injection, and the locally highly-sheared flows associated with internal and edge transport barriers in tokamaks.

In Section 2 we present an overview of the confinement required for NCSX; this is assessed using a 0-D model with fixed profile shapes based on the profile predictions of a power balance code (see Section 3). The global confinement scaling assessment is supplemented with

temperature profile predictions based on solutions of the power balance equations using a combination of neoclassical and anomalous transport; these calculations are described in Section 3. The power balance analysis finds that the NCSX design point is 'neoclassically accessible' and that the energy transport due to field ripple is much smaller than the axisymmetric neoclassical transport. The predicted pressure profile shapes are within the range used in MHD stability studies [5].

\section{Experimental Basis for Projected Confinement of NCSX}

There are several ways to estimate the confinement of a new configuration. Empirical global scaling relations summarize a wide body of experience, but their extrapolability to a new type of device is unclear. Neoclassical transport theory is expected to set a lower bound on 
transport, and the core region of many stellarator experimental plasmas is at, or near, this lower bound. There are no widely validated models for anomalous transport in stellarators, so the anomalous transport models we use in Section 3 are described there.

\subsection{Global Energy Confinement Scalings in Stellarators and Tokamaks}

NCSX is a stellarator (with a fully 3-D magnetic configuration and stellarator-like radial profile of rotational transform), but its quasi-axisymmetric character suggests that the transport may be tokamak-like. That is, the neoclassical ripple transport is expected to be much smaller than the neoclassical axisymmetric transport, and the consequently much-reduced rippledamping of flows may allow zonal flows and neutral beam driven sheared flows to play an important role in suppressing anomalous transport. It is appropriate, therefore, to compare the anticipated energy confinement to standard scalings for both stellarators and tokamaks.

The ISS-95 [2] global confinement scaling represents typical confinement in unoptimized stellarators with H-mode discharges excluded. This scaling is based on the 'diamagnetic' stored energy ( total stored energy, including fast ions) and the estimated actual heating power (not the injected power), so we use the corresponding quantities in assessing $\mathrm{H}_{\mathrm{ISs}-95}$ for NCSX. The fast ion contribution to the stored energy can reach $\sim 25-30 \%$ of the total in low collisionality $<\square=4 \%$ NCSX plasmas, which is similar to the fast ion stored energy fraction in neutral beam heated, low density stellarator (and tokamak) plasmas. 
Plasmas heated by neutral beam injection and by RF waves at the electron cyclotron resonance are included in the ISS-95 database. No confinement difference has been linked to the heating method, but in the database the heating method and density are systematically correlated (higher density plasmas are heated predominantly by NBI). There is a further correlation between density and heating power: they rise together; it was possible to determine separate dependences on density and heating power for only some of the devices.

Many NCSX parameters lie within the range of the stellarator experiments that make up the ISS-95 database. The exceptions are the minor radius, NCSX is nearly $20 \%$ larger than ATF, the aspect ratio, which is lower than that of $\mathrm{CHS}$, and the heating power, at $6 \mathrm{MW}$, is twice the maximum in the ISS-95 database.

Subsequent to the development of the ISS-95 scaling, LHD [6, 7] has provided confinement data for a much larger stellarator with a minor radius up to twice the size of NCSX (and 10 times its volume), and with slightly more heating power than planned for NCSX. Energy confinement in LHD has ranged beyond twice the ISS-95 expectation $[8,9]$ even at high $<\square>[7$, 10], and has no apparent dependence on heating method [11]. The enhanced confinement is attributed to an edge temperature pedestal $[12,13,14]$, which is not generally observed in other stellarators but is not attributed to an H-mode in LHD. The pedestal may be associated with a chain of magnetic islands at or near the plasma edge [12], while avoidance of island chains at low order rational $\mathrm{t}$ - values is associated with $\mathrm{H}$-modes in W7-AS $[15,16]$. 
Many tokamaks contributed to the database which forms the foundation of the ITER-97P L-mode energy confinement scaling [3], which fits each tokamak individually as well, or better than, the earlier ITER-89P scaling [17]. Again, most parameter ranges (e.g., size, aspect ratio, $\mathrm{B}_{\mathrm{o}}$, density, $\mathrm{P}_{\text {heat }},<\square>$ ) encompass the NCSX design point. The exceptions are that $\mathrm{q}(\mathrm{a})$ is lower in NCSX and its magnetic shear is 'reversed' relative to typical tokamak shear. In evaluating $\mathrm{H}_{\text {ITER- }}$ ${ }_{97 \mathrm{P}}$ for NCSX we use an effective plasma current, $\mathrm{I}_{\mathrm{p}}{ }^{\text {eff }}$, which produces the same $\mathrm{q}(\mathrm{a})$ in an equivalent axisymmetric configuration: $\mathrm{I}_{\mathrm{p}}^{\text {eff }}=0.48 \mathrm{MA}\left(\mathrm{B}_{\mathrm{o}} / 1.2 \mathrm{~T}\right)$. See Section 2 for a description of how the effective plasma current and elongation are determined for NCSX.

The NCSX goal of $\left\langle\square>=4 \%\right.$ with $\mathrm{P}_{\text {heat }} \sim 4 \mathrm{MW}\left(\mathrm{R}_{\mathrm{o}}=1.4 \mathrm{~m}\right.$, and $\mathrm{B}_{\mathrm{o}}=1.2 \mathrm{~T}$ with the CDR configuration) can be achieved with $\mathrm{H}_{\text {ISS-95 }}=1.8$ and $\mathrm{H}_{\text {ITER-97P }}=0.7$ in a collisional, high-density plasma. (see Section 2). With an additional constraint that the minimum $\square_{i}^{*}=0.25$, the required $\mathrm{H}$ factors are raised: $\mathrm{H}_{\text {ISS-95 }}$ to 2.9 and $\mathrm{H}_{\text {ITER-97P }}$ to 0.9 (see Section 2). The required confinement is slightly better than has been achieved to date in unoptimized stellarators. LHD has a number of nearly steady state discharges with $<\square>2 \%$ and $\mathrm{H}_{\mathrm{ISS}-95}$ up to $2.0\left(\mathrm{H}_{\mathrm{ISS}-95}\right.$ rises to 2.4 with $\mathrm{dW} / \mathrm{dt} \sim$ $\left.0.13 \mathrm{P}_{\mathrm{abs}}\right)$. More recently, LHD has achieved $<\square>=4 \%$ [18]. W7-AS reports $\mathrm{H}_{\mathrm{ISS}-95}$ up to 2.5 [19, 20], and in other plasmas $\langle\square>=3 \%$ [21]. NCSX is similar in size to the PBX-M tokamak, which achieved $\left\langle\square>=6.8 \%\right.$ at $\mathrm{B}_{\mathrm{o}}=1.1 \mathrm{~T}$ with $5.5 \mathrm{MW}$ of neutral beam heating at $\mathrm{H}_{\text {ITER-97P }}=1.7$ and an estimated $\mathrm{H}_{\mathrm{ISS}-95} \sim 3.9$ [22].

The ISS-04 scaling [23] is based on an extension of the ISS-95 database that adds LHD and TJII in as well as much new data from W7-AS. The parametric dependences are little changed but the choice of overall normalization is different, and this leads to a different enchancement factor 
for the NCSX standard plasma: $\mathrm{H}_{\mathrm{ISS}-04} \sim 1.7$, which is at the upper boundary of confinement in the new database.

\subsection{Neoclassical Transport in Stellarators: Theory and Experiment}

Neoclassical theory sets a lower bound on the transport expected in NCSX, and provides another method of assessing the confinement required to achieve the design goals. In a number of instances the core regions of stellarator plasmas are in accord with neoclassical predictions, so these predictions are of more than academic interest. After a brief outline of some basic theoretical concepts concerning neoclassical transport in stellarators, some experimental results are summarized.

In the long-mean-free-path (lmfp) regime, particles localized within a stellarator's helical ripples often dominate the radial transport. For several orderings of the characteristic frequencies of the motion, analytic solutions of the bounce-averaged kinetic equation have been derived in several limiting cases. Each solution has a distinctive dependence on collisionality that is used to name these transport regimes. Of these stellarator-specific lmfp results, the $1 / \Pi$ regime is strongly influenced by the details of the magnetic-field geometry, whereas the magnitude of the radial electric field is more decisive in the $\sqrt{\square}$ and // regimes.

Neoclassical transport in a 3-D magnetic configuration is not intrinsically ambipolar, and any resulting net radial current sets up a radial electric field that changes the radial transport until the fluxes become ambipolar; this value of the radial electric field is referred to as the 'ambipolar $\mathrm{E}_{\mathrm{r}}$ '. There are generally at least two values of electric field which stably balance the radial 
particle fluxes: the so called 'ion root' usually has $\mathrm{E}_{\mathrm{r}}<0$ (mnemonically, $\mathrm{E}_{\mathrm{r}}$ 'pulls' the ions into the plasma), while the 'electron root', with $\mathrm{E}_{\mathrm{r}}>0$ ( $\mathrm{E}_{\mathrm{r}}$ 'pulls' the electrons into the plasma).

For typical magnitudes of the ambipolar radial electric field the ions are in the $\sqrt{\square}$ or $/ 1$ regimes, and the electrons are in the $1 / /$ regime. In the $1 / \square$ regime neoclassical energy transport due to helical ripple scales as $T^{9 / 2}$, and while ion transport is greatly reduced by the ambipolar $E_{r}$, stellarator plasmas often have temperatures below $2 \mathrm{keV}$. However, ion and electron temperatures up to $10 \mathrm{keV}$ have been observed in low density plasmas with electron cyclotron heating $[24,25]$. Neoclassical transport models based on analytic theoretical scalings, but normalized by numerical simulations, are typically used for analysis of experimental data.

As for comparison with experiment, the ambipolar radial electric field is reviewed first because the ion particle and energy transport depends strongly on $\mathrm{E}_{\mathrm{r}}$; if it differs from neoclassical expectations the transport fluxes will, too. The neoclassically predicted ambipolar radial electric field is generally in agreement with observations in the core of CHS [26] and W7AS $[27,28,29,30]$ in the 'ion root' regime. Agreement has also been obtained in plasmas with net toroidal current and significant magnetic shear in W7-AS [31], and qualitative agreement has been reported in TJ-II [32]. The general agreement even in plasmas with dominantly anomalous energy transport supports the widespread belief that anomalous transport is intrinsically ambipolar.

The frequent agreement with the neoclassically predicted electric field is very important because the neoclassical ripple transport is strongly dependent on $\mathrm{E}_{\mathrm{r}}$, particularly the ion channel. 
It may be possible to modify the radial electric field in NCSX with unbalanced momentum input from the neutral beams. This would allow enhancement of $E_{r}$ with little effect on overall transport, or reduction in order to confirm theoretical predictions of enhanced transport with small $\mathrm{E}_{\mathrm{r}}$.

In the theoretically predicted 'electron root' regime [33] the magnitude of $E_{r}$ is larger than in the 'ion root' (and $\mathrm{E}_{\mathrm{r}}$ changes sign), so the transport is correspondingly lower than with the ion root. The electron root would be especially valuable in reactors, since the ripple transport has strong temperature dependence. W7-AS experiments have confirmed that transport is reduced by either sign of $E_{r}$ [29]. The electron root is frequently predicted, but often not observed in low-density ECRH plasmas in W7-AS [29] and CHS [34]. We adopt ion root solutions in Section 3 because we have no reason to expect NCSX to be in the electron root regime.

Realization of the electron root regime was experimentally elusive, but it has been achieved in CHS [35, 36, 37], W7-AS [31, 38, 39] and LHD [40, 41, 42]; see also the recent review in Ref. 43. The results in Ref. 39 and 40 are of special interest because there is no nondiffusive electron flux involved. In all cases the reduction in transport is less than expected from naive application of neoclassical theory; in many cases this may be due to a non-diffusive flux of ripple trapped suprathermal electrons (driven by the ECRH absorption) that is outside the standard theoretical treatments and transports substantial energy [38]. The neoclassically driven $\mathrm{E}_{\mathrm{r}}$ may also reduce anomalous transport as $\mathrm{E}_{\mathrm{r}}$ driven by other means does in tokamaks, but this has only scanty support in stellarators [44]. 
In addition to confirmation of the predicted radial electric field, neoclassically predicted ion and electron energy transport is also frequently observed in the core of W7-AS plasmas [28, 29, 31, 45], and the core of ECRF heated CHS plasmas [34]. The transport due to ripple usually exceeds the axisymmetric component in existing stellarators [46, 34]. Neoclassical transport is also not dominant in the core of neutral beam heated LHD plasmas [9, 14, 47]. Transport is greater than neoclassical predictions, however, in the edge of W7-AS and ECRF heated CHS plasmas. Even the core of neutral beam heated CHS plasmas is usually anomalous [34, 14, 9], but ion confinement in the core of CHS is approximately neoclassical in the hot-ion mode [48] and the N-ITB mode [36]. ATF reported anomalous electron energy transport even in the plasma core $[49,50]$, while observing the neoclassically predicted bootstrap current [51]. The anomalous transport in ATF was attributed to dissipative, trapped-electron mode turbulence, but a transport model based on a combination DTE and ion temperature gradient modes does not explain the CHS data [34]. No model for anomalous transport is supported by data from several stellarators.

W7-AS has achieved up to $\mathrm{H}_{\mathrm{ISS}-95} \sim 2.5$ [20], but these plasmas are consistent with neoclassical predictions for $\mathrm{r} / \mathrm{a}<0.7$, and the 'ambipolar $\mathrm{E}_{\mathrm{r}}$ ' is consistent with the measurements at all radii [29]. The unusually 'narrow' density profile (associated with the low-recycling conditions needed for this high confinement regime) is a key to the enhanced confinement, but not a departure from neoclassical behavior because the steeper density gradient leads to higher electric fields. The more recently discovered regime of enhanced confinement in W7-AS at very high density does not have narrow density profiles [21], and the temperatures are too low to produce significant ripple transport so anomalous transport is believed to be dominant here. 
Energy transport in dimensionless scaling experiments with matched CHS and LHD plasmas (with significant anomalous contributions in both devices) found that core transport follows gyro-Bohm scaling and the outer regions had scaling between Bohm and gyro-Bohm [14,9]. In several cases the anomalous contribution to the ion energy transport in LHD is close to or smaller than the expected neoclassical contribution [14,9], but it is always non-negligible.

In both CHS [26] and LHD [9, 47] 'inward shifted' configurations with slightly smaller $R_{0}$ have somewhat improved energy confinement relative to the standard $R_{0}$. The improvement is attributed to smaller neoclassical orbit drifts in these inward shifted configurations, but anomalous transport is significant in the core for all $R_{o}$ in both devices, and becomes small relative to neoclassical only for outward shifted LHD configurations with strongly de-optimized drift orbits and enhanced neoclassical ripple transport.

Generally speaking, neoclassical theory is reliable for predicting the radial electric field in stellarators (false predictions of the 'electron root' are a notable exception), and the resulting reduction in neoclassical ripple transport (from its level with $\mathrm{E}_{\mathrm{r}}=0$ ) has been widely validated. Neoclassical energy transport is often dominant in the core of lower density plasmas in W7-AS, but anomalous energy transport is usually significant or even dominant in the outer plasma of all stellarators and in the core of CHS and LHD. Unfortunately, there is no physical understanding of anomalous transport in stellarators that might indicate the conditions where it will be important. 


\subsection{Enhanced Confinement Regimes in Stellarators}

A number of enhanced confinement regimes have been reported in stellarators; some of these appear to be similar to tokamak regimes, and the H-mode is among them [52]. In stellarator H-modes the energy confinement is enhanced modestly (no more than $30 \%$ ) in W7-AS $[15,16]$ and CHS $[53,54,55,56]$. Access is typically restricted to narrow ranges in $\boxminus$ that minimize damping of poloidal rotation at the plasma boundary by avoiding island chains $[57,58]$ (in W7AS toroidal rotation is strongly damped by toroidal mirrors, but poloidal rotation is possible).

Internal transport barriers are also widely reported in stellarators [59]. CHS reports two enhanced confinement regimes associated with a change in the radial electric field. One is a 'high ion temperature mode' [48] said to be similar to TFTR supershots and hot ion modes in JET and JT-60U; the similarity includes a sustained peaked density profile, which is rare in stellarators. The other enhanced confinement regime involves electron root suppression of neoclassical transport that has been named N-ITB for 'neoclassical internal transport barrier'. These plasmas can be steady state [36], or have a dynamic $\mathrm{E}_{\mathrm{r}}$ bifurcation character $[60,61,62]$ with rapid variations in the magnitude of the radial electric field driven by central ECH; these appear to confirm theoretical predictions of $\mathrm{E}_{\mathrm{r}}$ bifurcation [63].

As described above, W7-AS reports two distinct regimes of enhanced confinement. One is characterized as a low-recycling regime with 'narrow' density profiles and $\mathrm{H}_{\mathrm{ISS}-95}$ up to 2.5 [19, 20]. The other is a very high-density regime with broad density profiles and $\mathrm{H}_{\mathrm{ISS}-95}$ up to 2 [21]. 
LHD confinement is generally enhanced, with $\mathrm{H}_{\text {ISs-95 }}$ up to 2 , when operated in an inward shifted configuration $[8,9,47]$. Perhaps this is a special confinement regime associated with the unusually high peripheral temperatures in LHD, or with reduced neoclassical transport (particularly the viscosity?) [47], or perhaps it is an indication that the ISS-95 scaling should be adjusted to better fit this new data set which lies outside the parameter range of the ISS-95 database. This last idea is not supported by analysis of the ISS-04 database: the inward shifted LHD plasmas systematically have improved confinement relative to the overall parametric scaling which best fits the combined data set that includes LHD data.

The detailed reasons for enhanced confinement in toroidal devices are not fully understood so it is difficult to predict what will occur in any new device. The overall lessen to be drawn is that there are apparently a number of causes of enhanced confinement, and perhaps some of them will be operative in NCSX. In addition to the above causes, the magnetic shear in NCSX is similar to that of 'reversed shear' enhanced-confinement regimes in tokamaks, and this leads to a reversal of trapped-particle drift precession that should stabilize trapped-particle driven microinstabilities [64].

Standard techniques for confinement enhancement in stellarators and tokamaks are planned for NCSX. These include wall conditioning, control of wall recycling, unbalanced neutral beam injection, pellet injection, limiting regions of high flux expansion, and edge biasing. 


\section{Global Scaling Model: Plasma Operation Contours}

A 0-D model is used to illustrate the relationship between several important plasma parameters in Figure 1. Contours of the minimum ion collisionality and confinement $\mathrm{H}$ factors required for a given $<\square>$ and density are shown for two neutral beam power levels. To increase reactor-relevance it is desirable to test transport, stability, and bootstrap current at moderately low collisionality, but raising the density - and collisionality - reduces the value of $\mathrm{H}_{\mathrm{ISS}-95}$ needed to reach a given $<\square>$.

The global scaling model assumes $\mathrm{R}_{\mathrm{o}}=1.4 \mathrm{~m}, \mathrm{~B}_{\mathrm{o}}=1.2 \mathrm{~T}$, and fixed profile shapes for density and temperature (those in Figure 5; note that the minimum ion collisionality is located at $\mathrm{r} \sim 0.7 \mathrm{a})$. The maximum density in the figure is at the Sudo density limit [65] for stellarators. The 0-D model is comprised of the following equations (powers are in MW, stored energy in $\mathrm{MJ}, \mathrm{B}_{\mathrm{o}}$ in Tesla, a and $\mathrm{R}_{\mathrm{o}}$ in meters, $<\square>$ is not in $\%, \bar{n}_{e}$ is in $10^{19} / \mathrm{m}^{3}$ ):

Sudo density limit $=\left\{\mathrm{P}_{\text {heat }} \mathrm{B}_{\mathrm{o}} /\left(\mathrm{R}_{\mathrm{o}} \mathrm{a}^{2}\right)\right\}^{0.5} 2.5 \times 10^{19} / \mathrm{m}^{3}$

Based on Figure 5, the minimum $\square^{*}=0.027\left\{\bar{n}_{e}{ }^{3} \mathrm{R}_{\mathrm{o}} /(100<\square>)^{2} \mathrm{~B}_{\mathrm{o}}{ }^{4}\right\}$

The stored energy (in MJ) is $\mathrm{W}_{\text {tot }}=1.5<\square>\left(10 \mathrm{~B}_{\mathrm{o}}{ }^{2} / 2 \square\right) \mathrm{V}_{\mathrm{p}}$, where $\mathrm{V}_{\mathrm{p}}=2 \mathrm{R}_{\mathrm{o}}(\square \mathrm{a})^{2}$

The energy confinement time is $\square_{\mathrm{E}}=\mathrm{W}_{\text {tot }} / \mathrm{P}_{\text {heat }}$, and the $\mathrm{H}$ factors are

$\mathrm{H}_{\mathrm{ISS}-95}=\square_{\mathrm{E}} / \mathrm{a}^{2.21} \mathrm{R}_{\mathrm{o}}^{0.65} \mathrm{P}_{\text {heat }}{ }^{-0.59} \bar{n}_{e}{ }^{0.51} \mathrm{~B}_{\mathrm{o}}{ }^{0.83} \mathrm{E}(2 \mathrm{a} / 3)^{0.43} 0.079 \mathrm{sec}$

For hydrogen plasmas $\left(\mathrm{M}_{\mathrm{eff}}=1\right)$, the multiplier on the ITER-97P tokamak L-mode scaling is $\mathrm{H}_{\text {ITER-97P }}=\left(\mathrm{W}_{\text {tot }} / \mathrm{P}_{\text {inj }}\right) /(\mathrm{a} / \square)^{0.31} \mathrm{R}_{\mathrm{o}}{ }^{1.38} \square^{0.67} \mathrm{P}_{\text {inj }}{ }^{-0.57} \bar{n}_{e}{ }^{0.24} \mathrm{~B}_{\mathrm{o}}{ }^{0.2} \mathrm{I}_{\mathrm{p}}^{0.74} 0.037 \mathrm{sec}$, 
where " $\mathrm{a} / \mathrm{\square}$ " represents the tokamak definition of minor radius; the symbol "a" follows the stellarator definition, which is derived from the volume enclosed by a magnetic surface, so "a/ $\square$ " is approximately the toroidally averaged midplane minor radius. The elongation we use for NCSX $(\square=1.77)$ is derived from the $n=0$ components of the spectral representation of the NCSX boundary.

The total plasma current is used in the ITER-97P confinement scaling, but using the internal current for any current free stellarator would lead to absurdly low predictions of energy confinement so a definition based on the rotational transform is more physically meaningful. The effective plasma current we use is the total plasma current in an axisymmetric configuration with a boundary given by the $\mathrm{n}=0$ components of the spectral representation of the NCSX boundary, with the same the rotational transform and pressure profiles as in the NCSX standard configuration. This effective plasma current scales with toroidal magnetic field as: $\mathrm{Ip}=\left(\mathrm{B}_{\mathrm{o}} / 1.2 \mathrm{~T}\right) 0.48 \mathrm{MA}$.

Using the total effective current, the ITER-97P scaling gives higher confinement times than are measured in the ISS-95 devices and LHD, but there is no reason to expect a tokamak scaling to be accurate for those devices because they are not quasi-axisymmetric. The energy confinement of L-mode plasmas in many tokamaks is approximately given [2] by the ISS-95 scaling when using an estimate of the rotational transform in tokamaks at $r / a=2 / 3$ (which is required by the ISS-95 scaling). One might then estimate the effective plasma current of stellarators at the location $\mathrm{r} / \mathrm{a}=2 / 3$ in a comparison of confinement to a tokamak $\mathrm{L}$-mode scaling, but this would be only the part of the effective plasma current that is inside $\mathrm{r} / \mathrm{a}=2 / 3$, not the total plasma current which is actually used in tokamak scalings. If the effective plasma current of NCSX were 
evaluated at $\mathrm{r} / \mathrm{a}=2 / 3$, then the required $\mathrm{H}$-factor based on this new scaling would be twice as high as those we are reporting.

The approximation for neutral beam orbit losses is based on the $B_{o}$ and $R_{o}$ scans in Ref. 66, which used the plasma parameters of Figure 5:

$\mathrm{P}_{\text {heat }}=\mathrm{P}_{\text {inj }}\left(1-0.24 / \operatorname{sqrt}\left\{\left(\mathrm{B}_{\mathrm{o}} / 1.2 \mathrm{~T}\right)\left(\mathrm{R}_{\mathrm{o}} / 1.4 \mathrm{~m}\right)\right\}\right)$

Higher density, colder plasmas (as in Figure 6) will have less orbit loss because there is less time for stochastic diffusion and because the slowing down rate is increased more than the pitch angle scattering rate; no 'credit' for this is taken in the expression for the orbit loss that is used here.

As $\langle\square>$ is raised at a fixed density in Figure 1 the stored energy and $\mathrm{H}$ factors rise linearly with $\left\langle\square>\right.$, and the collisionality drops as $1 /\langle\square\rangle^{2}$. The density dependence of the ISS-95 scaling lowers $\mathrm{H}_{\text {ISS-95 }}$ as the density rises, but the collisionality increases as $\bar{n}_{e}{ }^{3}$ at fixed $<\square>$. The maximum value on the density axis corresponds to the Sudo density limit.

Using the maximum injection power of $6 \mathrm{MW}$ minimizes the confinement required to reach a given condition. To reach $\langle\square\rangle=4 \%$ and $\square_{\square}^{*}=0.25$ requires $\mathrm{H}_{\mathrm{ISS}-95}=2.9$ and $\mathrm{H}_{\text {ITER-97P }}=0.9$. Raising the density to the Sudo limit lowers $\mathrm{H}_{\text {ISS-95 }}$ to 1.8 at $\left\langle\square>=4 \%\right.$, but then $\square_{\square}^{*}>1$ and the results are less relevant to subsequent devices. Finally, with confinement at the level of $\mathrm{H}_{\mathrm{ISS}-95}=1$, the achievable $<\square>$ is $\sim 2.2 \%$ at the Sudo density limit. Note that $\mathrm{H}_{\text {ITER-97P }}$ is below 1 for $\langle\square>=4 \%$ and $\square^{*}=0.25$, and is even lower for the other conditions described here. 
With the initial capability of $3 \mathrm{MW}$ injected, $\left\langle\square>\sim 3.3 \%\right.$ and $\square_{\square}^{*}=0.25$ can be achieved with $\mathrm{H}_{\text {ITER-97P }}=1.0$, and $\mathrm{H}_{\text {ISS-95 }}=2.9$ is compatible with $\left\langle\square>\sim 2.6 \%\right.$ and $\square_{\square}^{*}=0.25$ (this $\mathrm{H}_{\text {ISS-95 }}$ is sufficient for $\left\langle\square>=4 \%\right.$ and $\square^{*}=0.25$ with $6 \mathrm{MW}$ of heating). And finally, with confinement at the level of $\mathrm{H}_{\text {ISS-95 }}=1$, the highest $<\square>$ is $\sim 1.4 \%$ at the Sudo density limit.

As shown in Ref. 5, the independently powered modular coils provide sufficient shaping flexibility to vary the MHD stability limit down to $1 \%$. It is therefore possible to begin testing MHD stability predictions early with $3 \mathrm{MW}$ at $\mathrm{H}_{\text {ISS-95 }} \sim 1.5$; if confinement is at the level of $\mathrm{H}_{\text {ISS- }}$ ${ }_{95} \sim 1$ the full beam power of $6 \mathrm{MW}$ is sufficient to demonstrate stability at $\langle\square\rangle=2.2 \%$. Higher levels of confinement or heating power ( 6-8 MW of RF power could be added) are needed to reach the more interesting $\langle\square>$ limits of $4 \%$ or greater, but this is a plausible target since existing unoptimized stellarators reach $\mathrm{H}_{\mathrm{IS}-95} \sim 2-2.5$.

The central temperature in this space is indicated in Figure 2; the relationship between $<\square>$, density, and central temperature is based on the profile shapes in Figure 5. The contours in this figure are correct for any heating power, but the maximum on the density axis is the Sudo limit for $6 \mathrm{MW}$. Note that with high densities the temperature is generally below $1 \mathrm{keV}$, and impurity radiation could easily become a serious problem because impurities would not be completely stripped of their electrons. 


\section{Radial Power Balance Calculations for NCSX}

One of the NCSX design goals is to greatly reduce transport due to helical ripple by producing a highly quasi-axisymmetric configuration. With low 'effective' helical ripple the neoclassical particle, energy, and momentum transport should all be reduced relative to unoptimized stellarator designs, and may be similar to tokamak transport. Anomalous transport may also be reduced by the effects of flow shear as well as the magnetic shear - which is similar to 'reversed shear' in tokamaks, where it can lead to enhanced confinement.

The temperature predictions described in this section employ the self-consistent radial electric field, $E_{r}$, which would be set up by the neoclassical ripple transport. The ion energy and particle transport are strongly dependent on $\mathrm{E}_{\mathrm{r}}$, so the self-consistent value must be used, i.e., the value that produces ambipolar fluxes. With this ambipolar $\mathrm{E}_{\mathrm{r}}$ the DKES and MOCA codes confirm that the neoclassical transport approaches the axisymmetric level (see Section 3.2).

\subsection{Radial Power Balance Methodology with Analytic Transport Model}

Self-consistent temperature profile predictions involve several steps:

1) estimate the $E_{r}$ necessary for ambipolar particle flux,

2) estimate the ripple, axisymmetric, and anomalous transport diffusivities,

3) predict temperature profiles, and then iterate all three steps until convergence occurs. The temperature profile predictions are solutions of the coupled, steady-state power balance equations for the electron and ion energy fluxes. The thermal diffusivities are made up of three 
parts: neoclassical ripple transport, neoclassical axisymmetric transport, and an anomalous transport model with an adjustable coefficient.

The neoclassical ripple transport depends on the density and temperature - and their gradients - as well as the radial electric field that, in turn, depends on the ripple-induced particle transport. Everything must be solved for 'simultaneously', so an iterative procedure is used until the radial electric field, temperatures, and transport fluxes have converged. By construction, the algorithm for finding the ambipolar electric field searches for an ion root solution, and will search for an electron root solution only if an ion root is not found.

The analytic model for neoclassical ripple transport used here began with the ShaingHoulberg model [67, 63, and earlier references therein], which is based on asymptotic theory for two collisionality regimes $(1 / \square$ and //) in a 'single helicity' magnetic configuration. Crume, et al., [68] improved the model for the // regime (this improvement was confirmed in Ref. 69), and the $1 / \square$ regime was changed to more accurately reflect the original asymptotic theory and to use an effective helical ripple described below. The resulting model is described in detail in Ref. 34. Neoclassical ripple transport is quite unimportant in our NCSX simulations so the improvements to the model have had only a small effect on the predictions.

Mynick [70] used a Monte Carlo transport simulation to approximately verify related transport expressions for a single helicity magnetic configuration, and Beidler has also verified that numerical simulations of neoclassical transport exhibit the expected collisionality dependences in a number of stellarator configurations [69,71, 72,], although the // regime has 
not been identified in some configurations [73]. It is vital to note, however, that the theoretical normalization of these scalings is not correct even for simple model magnetic configurations (see Ref. 69 for details), and accurate normalization must be based on numerical transport calculations for specific magnetic configurations.

The single helicity analytic model has been extended in the $1 / \square$ regime to more complex magnetic configurations [74] and benchmarked against two other calculation methods [73] (more complete discussion is given in the next section). The effective helical ripple for NCSX is shown in Figure 3. For comparison we note that the effective helical ripple of $\mathrm{W} 7-\mathrm{X}$ is close to 0.01 at all radii, and that of ATF ranged from 0.3 near the edge to $\sim 0.1$ deep in the core.

The presence of plateau (the same regime found in neoclassical transport theory for tokamaks), $1 / \Pi$ and $\sqrt{\square}$ regimes has been confirmed in calculations of the radial transport coefficient in all stellarator configurations. A / / scaling is also indicated at the lowest collisionalities in some devices, including NCSX. This suggests that a compact, yet accurate, semi-analytical description of radial transport coefficients remains a realistic possibility, although an efficient method of obtaining the fitted coefficients for new variations of a magnetic configuration remains to be found. Such methods are currently being developed.

The neoclassical axisymmetric transport is given by the Chang-Hinton [75] formulation for a circular plasma cross section with the same cross sectional area as the toroidal average of NCSX; the implementation was adopted from SNAP [76]. A an axisymmetric calculation by NCLASS [77], using the non-circular toroidal average shape of NCSX and profiles close to those 
in Figure 5, shows that the Chang-Hinton formulation of axisymmetric transport should be reduced by $35 \%$ for these conditions, so this correction factor was used in the calculation of the profiles in Figure 5. Other collisionality regimes, such as that in Figure 6, may require a different correction; no correction was used for Figure 6.

There are no empirical transport models that are broadly supported by experimental data, so the anomalous diffusivity in the predictions is adjusted to match a target thermal $<\square>$ or $\mathrm{H}$ factor. Comparing the resulting anomalous conduction power to the neoclassical conduction power provides a measure of confinement 'robustness'. Errors in the neoclassical models will therefore cause errors in the degree of robustness and minor changes in profile shapes, but not the overall temperature magnitudes because these are determined by the target thermal $<\square>$ or $\mathrm{H}$ factor. Stellarators often have experimentally determined thermal diffusivities that are approximately radially constant (unlike many tokamaks), so our simplest anomalous transport model is spatially uniform. Alternatively, a radially varying Lackner-Gottardi expression for anomalous transport can also be used. This anomalous transport model,

$$
\square^{\mathrm{LG}}=1.5 \mathrm{~m}^{2} / \mathrm{s}\left(1.6 \mathrm{~m} / \mathrm{R}_{\mathrm{o}}\right)\left(2 \mathrm{~T} / \mathrm{B}_{\mathrm{o}}\right)^{2} \mathrm{~T}_{\mathrm{e}, \mathrm{keV}}{ }^{1.5} /\left(1.1-(\mathrm{r} / \mathrm{a})^{2}\right)^{4},
$$

is based on one originally developed for ASDEX [78], with additional $\mathrm{B}_{\mathrm{o}}$ and $\mathrm{R}_{\mathrm{o}}$ scaling inspired by the Lackner-Gottardi global scaling [79], and its use in a simulation of one W7-AS discharge has been reported [80]. The anomalous transport multiplier used here is $<<1$ and the same for the electron and ion diffusivities, but temperature equilibration tightly couples the temperatures so only the temperatures near the edge are strongly affected if the anomalous transport is assigned 
to only one species. This Lackner-Gottardi diffusivity increases strongly in the outer region of the plasma; as a result it is the dominant term only near the edge and its contribution is insignificant in the plasma core with the multipliers needed to reach the NCSX target plasmas (see Figure 5).

The power balance solver described above has been used in TRANSP to develop the discharge evolution described in Ref. 81. The anomalous diffusivity was dynamically adjusted to meet a target $\square_{E}$ that was the lesser of the L-mode scaling and neo-Alcator scaling [82]; the latter applies in the low-power ohmically heated phase of the discharge when the L-mode scaling predicts relatively large $\square$.

The 'triangular' heating profile used here is similar to those calculated by TRANSP in Ref. 81 for neutral beam injection into an axisymmetric torus with the oblate portion of the NCSX cross section. The orbit loss calculations in Ref. 66 use the full 3-D geometry, and show relatively weak dependence on the injector's toroidal location, so the heating calculation in axisymmetric geometry may be a fair approximation to a 3-D heating calculation. The heating power is split between ions and electrons according to the Stix thermalization model [83].

The power balance equations are solved with an assumed density profile shape (see Figure 5c), and assumed outer boundary temperatures. The resulting temperature profiles are not sensitive to variations in these assumptions, largely because the anomalous transport multiplier is adjusted until the predictions match a target (typically $<\square>=4 \%$ ). Where the anomalous multiplier goes to zero defines the boundary of the 'neoclassically accessible' region. For a $\mathrm{B}_{\mathrm{o}}$ 
scan at $\langle\square>=4 \%$ the neoclassical boundary is above the maximum magnetic field of the current $\mathrm{R}_{\mathrm{o}}=1.4 \mathrm{~m}$ design.

\subsection{Neoclassical Transport: Analytic Theory vs. Numerical Simulations}

In several important ways real 3-D magnetic configurations more complex than those assumed in developing analytic neoclassical transport models. Real stellarators have poloidally varying helical field magnitude as well as multiple helicity components, and in some stellarators there is no single dominant component. In particular, many components make significant contributions in quasi-axisymmetric configurations. In complex configurations there will generally be multiple trapped particle populations, and each can have transport 'resonances' where the electric and magnetic contributions to the poloidal drift cancel (see Figure 1 of Ref. 45).

More complete neoclassical transport simulations are readily compared with each other and analytic theory by focusing on the most fundamental diffusivities. The local ansatz underlying neoclassical transport theory allows an ordering of the drift kinetic equation so that the minor-radius and energy coordinates appear only as parameters, reducing a nominally $5 \mathrm{D}$ problem to a more manageable 3D. It also becomes possible to characterize neoclassical effects in terms of three mono-energetic coefficients describing the radial transport, the bootstrap current, and the parallel conductivity. The full neoclassical transport matrix is subsequently obtained by appropriate convolutions of the mono-energetic coefficients with the Maxwellian particle distribution. 
To complement the special-case analytic solutions, there are two classes of numerical algorithm for solving the full drift kinetic equation in complex 3-D configurations: Monte Carlo particle orbit simulation, and basis function representation of the distribution function. These approaches have complementary shortcomings (problematic separation of the 'diffusive' and 'convective' contributions to the overall transport when the latter is present in the Monte Carlo simulations, and increasingly poor convergence of the basis function approach with decreasing collisionality), but they can be employed for arbitrarily complex magnetic fields and a wide range of collisionality. Their agreement in several benchmarks [73] lends confidence that these purely numerical simulations of transport have been correctly implemented.

The ability to handle arbitrarily complex magnetic fields is also a strength of the fieldline-integration technique [74]. Here, the drift kinetic equation is considerably simplified by assuming that the fast motion along field lines constitutes the only particle drift within a flux surface, ultimately allowing one to express the transport through the flux surface as a weighted integral of the geodesic curvature along a field line of 'infinite' length (i.e. sufficiently long to cover the entire magnetic surface). Evaluating the integral numerically provides an efficient means of determining the radial transport for any non-symmetric magnetic configuration in the $1 / \Pi$ regime. The validity of the results is confined to this regime, however, as a consequence of the assumption of negligible cross-field drift within a flux surface.

The most complete numerical simulations of neoclassical transport in multi-helicity magnetic geometry use Monte Carlo methods $[84,70,85,86]$ or are based on a drift kinetic 
equation solver, such as DKES $[87,88]$, or a ripple-averaged kinetic equation solver, GSRAKE [69]. All of these codes have been benchmarked with analytic theory in simple magnetic geometry [73]. Both classes of code have also exhibited the various analytic transport regimes in the mono-energetic diffusivities obtained for complex geometries $[86,89,73]$, although the coefficients are not predicted by analytic theory and must be fitted to the numerical results. However, the coefficient for the $1 / \square$ regime can be accurately evaluated for multi-helicity magnetic configurations by the NEO code [74], which provides an 'effective helical ripple' valid in the $1 / \square$ regime. Not all particle energies and species are in the $1 / \square$ regime, however, so we have carried out further calculations for NCSX to cover all collisionality regimes.

Mono-energetic transport coefficients calculated by the DKES $[87,88]$ code are shown in Figure 4 for $\mathrm{r}=0.5 \mathrm{a}$. In the low collisionality regime, the normalized particle transport coefficient, $\square_{11}{ }^{*}$, approaches the equivalent axisymmetric result as the electric field is increased; and the expected magnitude of $\mathrm{E}_{\mathrm{r}} / \mathrm{Bv}$ for ions is greater than the $3 \times 10^{-3}$ level shown in the figure. With the radial electric field required for ambipolar flux (as determined above) the transport will be very close to the axisymmetric result. The bootstrap coefficient, $\square_{31}$, is not far from the axisymmetric result (although the latter is not the limit for large $E_{r}$ ) and shows much less dependence on $\mathrm{E}_{\mathrm{r}}$ than is usual for stellarators but this $i$ typical of tokamaks. The Monte Carlo code MOCA [90] confirmed the DKES results and was able to extend them to lower collisionality [73] (where DKES convergence is problematic). 
The DKES and MOCA calculations thereby confirm that neoclassical ripple transport is expected to be insignificant in the planned NCSX conditions. This conclusion could conceivably become locally invalid if strong central electron heating were used in NCSX: the resulting high central electron temperatures would dramatically increase the ripple fluxes, but even in this case the ripple fluxes would be small in the cooler plasma outside the heated region.

\subsection{Temperature Projections for NCSX Scenarios}

Figure 5 shows plasma profiles for the 'standard' high $<\square>$ condition $\left(\mathrm{P}_{\mathrm{inj}}=6 \mathrm{MW}, \mathrm{B}_{\mathrm{o}}=1.2\right.$ $\mathrm{T}, \mathrm{R}_{\mathrm{o}}=1.4 \mathrm{~m}$ ), where the assumed density profile shape has been normalized so that the minimum $\square^{*}=0.25$ at this $<\square>$. Truly 'reactor relevant' collisionality values would require much higher $\mathrm{H}$ factors (or $\mathrm{B}_{\mathrm{o}}$, or $\mathrm{P}_{\mathrm{inj}}$ ), but this plasma is expected to be in the relevant collisionality regime from the point of view of energy transport and bootstrap current generation. The pressure profile is within the range used for MHD stability studies in Ref. 5. With the highly quasi-axisymmetric magnetic geometry the neoclassical ripple transport is negligible compared to the neoclassical axisymmetric transport, which has been normalized to an axisymmetric NCLASS [77] calculation for these conditions. Thus, the neoclassical transport is close to that of an equivalent tokamak. The radially constant anomalous transport diffusivity has been chosen to determine how much anomalous transport can be tolerated in the plasma core; we find that the anomalous transport exceeds the neoclassical transport in the outer $2 / 3$ of the plasma and the two are comparable in the core. The self-consistent ambipolar radial electric field is in the 'ion root' regime everywhere. (The collisionality scaling used in Section 2 is based on this figure.) 
When matching a target for $<\square>, \mathrm{H}_{\text {ISS-95 }}$, or $\mathrm{H}_{\text {ITER-97P }}$, the fast-ion stored energy is not calculated by the power balance code. In plasmas with minimum $\square_{*}^{*} \sim 0.25$ the fast ion stored energy is expected to be $\sim 25 \%$ of the total, so the target for the thermal $\langle\square>$ is $3 \%$. A separate calculation using the profiles of Figure 5 confirmed that the total $\langle\square\rangle$, including fast ions, is $4.1 \%$.

While the neutral beams heat ions preferentially (by a 60:40 ratio in the plasma core), the neoclassical losses are also larger in the ion channel so $T_{e}>T_{i}$ in the plasma center. At larger radii $\mathrm{T}_{\mathrm{e}}<\mathrm{T}_{\mathrm{i}}$ because we have assumed the electron and ion anomalous thermal diffusivities are equal and the electrons are dominantly heated through temperature equilibration with the ions. If all anomalous transport is assigned to one species, then it is the colder species in the region where anomalous transport is dominant.

The density dependence of the ISS-95 scaling favors operation at high density. Raising the density to the Sudo limit reduces the $\mathrm{H}_{\mathrm{ISS}-95}$ needed to reach $\langle\square>=4 \%$ from 2.9 to 1.8 ; an example of the resulting profiles is shown in Figure 6. Ripple transport is even more unimportant at these lower temperatures because it has stronger temperature dependence than axisymmetric transport. The Lackner-Gottardi transport model was used, so anomalous transport is important only in the outer part of the plasma. The thermal beta is fully $4 \%$; the fast ion contribution will be much lower in this colder, denser plasma. Note that the collisionality is now high, however. These results of the power balance code are similar to those given by the 0-D model in Section 2 .

If the anomalous transport is sufficiently high to reduce $\mathrm{H}_{\mathrm{ISS}-95}$ to 1 , then $6 \mathrm{MW}$ injected into a plasma at the Sudo density limit produces $\langle\square>=2.2 \%$ - enough for tests of the lower $\langle\square>$ 
limits with unoptimized shapes (see Ref. 5). At these colder temperatures the ripple transport is reduced and the Lackner-Gottardi anomalous model is dominant in the outer 30\% of the minor radius.

The initial complement of neutral beam injectors will generate $3 \mathrm{MW}$ and would be expected to produce lower $<\square>$ for a given $\mathrm{H}$ factor. Again choosing the density so that minimum $\square_{\square}^{*}=0.25$, we find that $\mathrm{H}_{\text {ISS-95 }}=2.9$ or $\mathrm{H}_{\text {ITER-97P }}=0.9$ implies $\langle\square>=2.5-2.8 \%$, respectively, at $\mathrm{B}_{\mathrm{o}}=1.2 \mathrm{~T}$. This is again consistent with the 0 -D results above, and would exceed the lower $<\square>$ limits which are predicted for less optimized shapes. 


\section{$4 \quad$ Summary}

We find that the high degree of quasi-axisymmetry of the NCSX configuration reduces the neoclassical helical ripple transport to a small fraction of the neoclassical axisymmetric transport in all tested NCSX configurations. (It is assumed here that the actual radial electric field - including any contribution driven by unbalanced neutral injection - reduces the ripple transport at least as much as the 'ambipolar' electric field would.) This means that neoclassical transport in NCSX is tokamak-like in the sense of being dominated by axisymmetric transport.

The very low effective ripple in NCSX greatly reduces the neoclassical viscosity [4], and this is expected to greatly reduce the rotation damping that is commonly observed in stellarators. This may be beneficial for reducing anomalous transport by permitting the development of zonal flows, large-scale sheared flows driven by neutral beam injection, and locally highly-sheared flows associated with internal and edge transport barriers. With balanced co and counter beams it will be possible to vary the external momentum input and, thus, the flow shear to study its effects on transport. The magnetic shear is similar to the 'reversed shear' enhanced-confinement regimes in tokamaks, and this provides another means of reducing anomalous transport to a low level.

Relative to the ISS-95 global energy confinement scaling, the confinement required to reach the $\langle\square>$ goal of $4 \%$, and low collisionality, simultaneously, is slightly higher than that already achieved in unoptimized stellarators. This level of confinement is $\sim 10 \%$ lower than 
predicted by the ITER-97P tokamak L-mode scaling. By operating near the Sudo density limit for stellarators, the required enhancement over the ISS-95 scaling is reduced by $35 \%$.

A combination of neoclassical and anomalous transport models predicts pressure profile shapes that are within the range of those used to study the MHD stability of NCSX. They also show that $\langle\square>=4 \%$ plasmas are 'neoclassically accessible', and can tolerate large levels of anomalous transport in the outer region of the plasma and comparable levels of neoclassical and anomalous transport in the core. Core temperatures of up to $\sim 2 \mathrm{keV}$ are expected in plasmas with moderate collisionality.

The initial complement of $3 \mathrm{MW}$ of neutral beam injection power will be sufficient to produce $\left\langle\square>=2.6-2.9 \%\right.$ at $\mathrm{B}_{0}=1.2 \mathrm{~T}$ (assuming $\mathrm{H}_{\text {ISS-95 }}=2.9$ or $\mathrm{H}_{\text {ITER-97P }}=0.9$ ) at moderate collisionality. This would be sufficient to test the lower $<\square>$ limits which are predicted for less optimized shapes (see Ref. 5).

\section{Acknowledgements}

It is a pleasure to acknowledge many useful discussions with A. Fujisawa, K. Ida, S. Murakami, N. Nakajima, J. Nuehrenberg, S. Okamura, H. Yamada, K. Yamazaki, and M. Yokoyama. This work was supported by DOE contract DE-AC02-76CH03073. The work of collaborators was partly supported by associations of EURATOM with CIEMAT, IPP and ÖAW, respectively. The content of the publication is the sole responsibility of its publishers and it does not necessarily represent the views of the Commission or its services. 


\section{Figure Captions}

Figure 1. Contours of $\mathrm{H}_{\mathrm{ISS}-95}$ (solid), $\mathrm{H}_{\mathrm{ITER}-97 \mathrm{P}}$ (dashed), and $\square_{i}^{*}$ (dotted) as functions of line average density and volume average beta; with $6 \mathrm{MW}$ of heating power on the left, $3 \mathrm{MW}$ on the right.

Figure 2. Contours of central temperature as functions of line average density and volume average beta for $B_{0}=1.2 \mathrm{~T}$; based on reference plasma shown in Figure 5 .

Figure 3. The effective helical ripple for NCSX vs the square root of the normalized toroidal flux; calculated by the NEO code.

Figure 4. DKES results for NCSX at $\mathrm{r}=\mathrm{a} / 2$ : (a) mono-energetic particle transport coefficient normalized to the plateau value of an equivalent elongated axisymmetric configuration, and (b) the normalized bootstrap current coefficient. The abscissa is the inverse of the mean free path. Radial electric field values: $\mathrm{E}_{\mathrm{r}} /(\mathrm{Bv})=0$ squares, $3 \times 10^{-4}$ filled diamonds, $1 \times 10^{-3}$ circles, $3 \times 10^{-3}$ empty diamonds, $1 \times 10^{-2}$ filled triangles, $3 \times 10^{-2}$ empty triangles. Dotted curves show the axisymmetric coefficients.

Figure 5. Profiles for a standard $\langle\square>=4 \%$ plasma: a) ion and electron temperatures, b) conducted power due to axisymmetric neoclassical transport, helical ripple neoclassical transport, and 'anomalous' transport which completes the power balance (radially flat anomalous diffusivity 
assume), c) electron density and ambipolar radial electric field, d) ion and electron collisionalities - normalized to the toroidal bounce frequency.

Figure 6. Profiles for a high density plasma with $\langle\square\rangle=4 \%$ : a) ion and electron temperatures, b) conducted power due to axisymmetric neoclassical transport, helical ripple neoclassical transport, and 'anomalous' transport which completes the power balance (Lackner-Gottardi form, but with reduced coefficient to match $\langle\square>=4 \%$ as explained in the text), c) electron density and ambipolar radial electric field, d) ion and electron collisionalities - normalized to the toroidal bounce frequency. 


\section{References}

[1] M C ZARNSTORFF, et al., "Physics of the compact advanced stellarator NCSX", Plasma Phys. Control. Fusion 43 (2001) A237-A249.

[2] U. STROTH, et al., "Energy Confinement Scaling from the International Stellarator Database", Nuclear Fusion 36 (1996) 1063.

[3] S. KAYE, et al., "ITER L-Mode Confinement Database”, Nuclear Fusion 37 (1997) 1303.

[4] A. REIMAN, et al., "Effect of ambipolar plasma flow on the penetration of resonant magnetic perturbations in a quasi-axisymmetric stellarator", Nucl. Fusion 45 (2005) 360.

[5] N. POMPHREY, et al., "NCSX Magnetic Configuration Flexibility and Robustness", submitted to Fusion Sci. Technol.

[6] A. IIYOSHI, et al., "Overview of the Large Helical Device project”, Nuclear Fusion 39 (1999) 1245.

[7] M. FUJIWARA, et al., “Overview of LHD experiments”, Nuclear Fusion 41 (2001) 1355.

[8] O. MOTOJIMA, et al., "Initial physics achievements of large helical device experiments", Phys. Plasmas, 6 (1999) 1843.

[9] H. YAMADA, et al., "Energy confinement and thermal transport characteristics of net current-free plasmas in the Large Helical Device”, Nuclear Fusion, 41 (2001) 901.

[10] S. SAKAKIBARA, et al., "MHD characteristics in the high beta regime of the Large Helical Device”, Nuclear Fusion, 41 (2001) 1177.

[11] T. WATARI, et al., “The performance of ICRF heated plasmas in LHD”, Nuclear Fusion, $41(2001) 325$.

[12] N. OHYABU, et al., "Thermal transport barrier in heliotron-type devices: Large Helical Device and Compact Helical System”, Phys. Plasmas, 7 (2000) 1802.69 
[13] K. NARIHARA, et al., "Observation of the "Self-Healing" of an Error Field Island in the Large Helical Device”, Phys. Rev. Letters, 87 (2001) 135002.

[14] H. YAMADA, et al., "Energy Confinement Time and Heat Transport in Initial Neutral Beam Heated Plasmas on the Large Helical Device”, Phys. Rev. Letters, 84 (2000) 1216. [15] V. ERCKMANN, et al., "H-mode of the W7-AS Stellarator", Phys. Rev. Letters, 70 (1993) 2086.

[16] M. HIRSCH, et al., "Operational range and transport barrier of the H-mode in the stellarator W7-AS”, Plasma Phys. Control. Fusion 40 (1998) 631.

[17] P. YUSHMANOV, et al., "Scalings for tokamak energy confinement", Nuclear Fusion 30 (1990) 1999.

[18] O. MOTOJIMA, et al., "Overview of confinement and MHD stability in the Large Helical Device", Nucl. Fusion 45 (2005) S255-S265..

[19] M. KICK, et al., "High ion temperatures and high beta in W7-AS", $16^{\text {th }}$ IAEA Fusion Energy Conference, Montreal, 2 (1996) 27.

[20] U. STROTH, et al., "High-confinement NBI discharges in the W7-AS stellarator", Plasma Phys. Control. Fusion 40 (1998) 1551.

[21] K. MCCORMICK, et al., "New Advanced Operational Regime on the W7-AS Stellarator", Phys. Rev. Letters, 89 (2002) 015001.

[22] N. SAUTHOFF, et al., "PBX-M research progress: approach to second stability", $13^{\text {th }}$ IAEA Fusion Energy Conference, Washington, 1 (1990) 709.

[23] H. YAMADA, et al., "Characterization of energy confinement in net-current free plasmas using the extended International Stellarator Database", Nucl. Fusion 45 (2005) 1684-1693. 
[24] S. KUBO, et al., "Extension and characteristics of an ECRH plasma in LHD", Plasma Phys. Control. Fusion 47 (2005) A81-A90.

[25] Y. TAKEIRI, et al., "High-ion temperature experiments with negative-ion-based neutral beam injection heating in Large Helical Device", Nucl. Fusion 45 (2005) 565-573.

[26] S. OKAMURA, et al., "Confinement physics study in a small low aspect ratio helical device: CHS”, Nuclear Fusion 39 (1999) 1337.

[27] H. MAASSBERG, et al., “Transport in Stellarators”, Plasma Phys. Control. Fusion 35 (1993) B319.

[28] J. BALDZUHN, et al., "Measurement and calculation of the radial electric field in the stellarator W7-AS”, Plasma Phys. Control. Fusion 40 (1998) 967.

[29] M. KICK, et al., "Electric field and transport in W7-AS”, Plasma Phys. Control. Fusion 41 (1999) A549.

[30] H. EHMLER, Y. TURKIN, C.D. BEIDLER, H. MAAßBERG, A. DINKLAGE, T. KLINGER and the W7-AS TEAM, "Experimental check of neoclassical predictions for the radial electric field in a stellarator", Nucl. Fusion 43 (2003) L11-L13.

[31] V. ERCKMANN, et al., "High density ECRH and shear related confinement with ECCD in W7-AS”, 16 $6^{\text {th }}$ IAEA Fusion Energy Conference, Montreal, 2 (1996) 119.

[32] E. ASCASIBAR, et al., "Confinement and stability on the TJ-II stellarator”, Plasma Physics and Controlled Fusion, 44 (2002) B307.

[33] H. E. MYNICK and W. N. G. HITCHON, "Effect of the ambipolar potential on stelllarator confinement”, Nuclear Fusion 23 (1983) 1053.

[34] K. YAMAZAKI and T. AMANO, "Plasma transport simulation modeling for helical confinement systems", Nuclear Fusion 32 (1992) 633. 
[35] A. FUJISAWA, et al., "Experimental study of plasma confinement and heating efficiency through potential profile measurements with a heavy ion beam probe in the Compact helical System", 16 ${ }^{\text {th }}$ IAEA Fusion Energy Conference, Montreal, 2 (1996) 41.

[36] T. MINAMI, et al., "Increased Understanding of Neoclassical Internal Barrier on CHS", $19^{\text {th }}$ IAEA Fusion Energy Conference, Lyon, 2002, EX/C4-3.

[37] T. MINAMI, et al., "Increased understanding of neoclassical internal transport barriers in CHS", Nucl. Fusion 44 (2004) 342-349.

[38] H. MAASSBERG, et al., "The neoclassical 'Electron Root' feature in the Wendelstein-7-AS stellarator", Phys. Plasmas, 7 (2000) 295.

[39] M ROMÉ, C D BEIDLER, H MAASSBERG, N B MARUSHCHENKO, YU A TURKIN and the W7-AS TEAM, "The 'electron-root' feature in the WENDELSTEIN-7-AS stellarator with ECRH in O1-mode compared to X2-mode", Plasma Phys. Control. Fusion 48 (2006) 353368.

[40] K. IDA, et al., "Reduction of Ion Thermal Diffusivity Associated with the Transition of the Radial Electric Field in Neutral-Beam-Heated Plasmas in the Large Helical Device", Phys. Rev. Letters, 86 (2001) 5297.

[41] K. IDA, et al., "Characteristics of Electron Heat Transport of Plasma with an Electron Internal-Transport Barrier in the Large Helical Device", Phys. Rev. Lett. 91(2003)085003-1. [42] T. SHIMOZUMA, et al., "Formation of electron internal transport barriers by highly localized electron cyclotron resonance heating in the large helical device", Plasma Phys. Control. Fusion 45 (2003) 1183-1192.

[43] M. YOKOYAMA, et al., "Common Features of Core 'Electron Root' Confinement in Helical Devices", submitted to Fusion Sci. Technol., ISW-15 issue. 
[44] A. FUJISAWA, et al., "Electron Thermal Transport Barrier and Density Fluctuation Reduction in a Toroidal Helical Plasma”, Phys. Rev. Letters, 82 (1999) 2669.

[45] H. MASSBERG, et al., "Experimental and neoclassical electron heat transport in the LMFP regime for the stellarators W7-A, L-2, and W7-AS”, Phys. Fluids, B 5 (1993) 3627.

[46] H. RENNER, et al., "Confinement properties of the 'advanced stellarator' Wendelstein W7AS", $13^{\text {th }}$ IAEA Fusion Energy Conference, Washington, 2 (1990) 439.

[47] S. MURAKAMI, et al., "Effect of Neoclassical Transport Optimization on Electron Heat Transport in the Low-collisionality LHD plasma", submitted to Fusion Sci. Technol. for ISW-15 issue.

[48] K. IDA, et al., "Transition from L mode to high ion temperature mode in CHS heliotron/torsatron plasmas”, Nuclear Fusion 39 (1999) 1649.

[49] M. MURAKAMI, et al., "Energy confinement and bootstrap current studies in the Advanced Toroidal Facility", 13 ${ }^{\text {th }}$ IAEA Fusion Energy Conference, Washington, 2 (1990) 455. [50] M. MURAKAMI, et al., "Recent results from the ATF torsatron”, Phys. Fluids, B 3 (1991) 2261.

[51] M. MURAKAMI, et al., "Bootstrap-current experiments in a toroidal plasma-confinement device”, Phys. Rev. Letters, 66 (1991) 707.

[52] F. WAGNER and U. STROTH, “Transport in toroidal devices - the experimentalist's view”, Plasma Phys. Control. Fusion 35 (1993) 1321.

[53] K. TOI, et al., "Formation of an H-mode-like transport barrier in the CHS heliotron/torsatron", 14 ${ }^{\text {th }}$ IAEA Fusion Energy Conference, Wurzburg, 2 (1992) 461. [54] K. TOI, et al., "H-mode transition in the CHS heliotrons/torsatron", Plasma Phys. Control. Fusion 36 (1994) A117. 
[55] K. TOI, et al., "Impact of rotational transform profile control on plasma confinement and stability in CHS”, 15 ${ }^{\text {th }}$ IAEA Fusion Energy Conference, Seville, 2 (1994) 331.

[56] K. TOI, et al., "Transition behaviour in the H-mode of the CHS heliotron/torsatron", Plasma Phys. Control. Fusion 38 (1996) 1289.

[57] M. HIRSCH, et al., "Operational conditions and characteristics of ELM-events during Hmode plasmas in the stellarator W7-AS”, Plasma Phys. Control. Fusion 42 (2000) A231.

[58] R. JAENICKE, et al., "Operational Boundaries on the Stellarator W7-AS at the Beginning of the Divertor Experiments", $18^{\text {th }}$ IAEA Fusion Energy Conference, Sorrento, 2000, OV4/3. [59] A. FUJISAWA, “Transport barriers and bifurcation characteristics in stellarators", Plasma Phys. Control. Fusion 45 (2003) R1.

[60] A. FUJISAWA, et al., "Dynamic Behavior of Potential in the Plasma Core of the CHS Heliotron/Torsatron”, Phys. Rev. Letters, 79 (1997) 1054.

[61] A. FUJISAWA, et al., "Experimental study of the bifurcation nature of the electrostatic potential of a toroidal helical plasma", Phys. Plasmas 7 (2000) $4152 .$.

[62] A. FUJISAWA, et al., "Observation of bifurcation properties of radial electric fields using a heavy ion beam probe”, Nuclear Fusion, 41 (2001) 575.

[63] D. E., HASTINGS, W. A. HOULBERG, and K.-C. SHAING, "The ambipolar electric field in stellarators", Nuclear Fusion 25 (1985) 445.

[64] M. YOKOYAMA, et al., "Drift Reversal Capability in Helical Systems", $19^{\text {th }}$ IAEA Fusion Energy Conference, Lyon, 2002, IC/P-08.

[65] S. SUDO, et al., "Scalings of energy confinement and density limit in stellarator/heliotron devices", Nuclear Fusion 30 (1990) 11. 
[66] H. KUGEL, et al., "Plasma Heating Methods", Fusion Sci. Technol., this issue.

[67] K.-C. SHAING, "Stability of the radial electric field in a nonaxisymmetric torus", Phys. Fluids 27 (1984) 1567.

[68] E. C. CRUME, JR, K. C. SHAING, S. P. HIRSHMAN, and W. I. VAN RIJ, “Transport scaling in the collisionless-detrapping regime in stellarators", Phys Fluids 31 (1988) 11.

[69] C. D. BEIDLER and W. D. D’HAESELEER, “A general solution of the ripple-averaged kinetic equations (GSRAKE)”, Plasma Phys. Control. Fusion 37 (1995) 463.

[70] H. E. MYNICK, "Verification of the classical theory of helical transport in stellarators", Phys. Fluids, 25 (1982) 325.

[71] C. D. BEIDLER, W. N. G. HITCHON, and J. L. SHOHET, “'Hybrid' Monte Carlo simulation of ripple transport in stellarators", J. Comp. Phys. 72 (1987) 220.

[72] C. D. BEIDLER and W. N. G. HITCHON, "Ripple transport in helical-axis advanced stellarators: a comparison with classical stellarators/torsatrons", Plasma Phys. Control. Fusion 36 (1994) 317.

[73] C. D. BEIDLER, et al., "Initial Results from an International Collaboration on Neoclassical Transport in Stellarators", $13^{\text {th }}$ Intl. Stellarator Workshop, Canberra, 2002, paper OI10. [74] V. V. NEMOV, et al., "Evaluation of $1 / \square$ neoclassical transport in stellarators", Phys. Plasmas, 6 (1999) 4622.

[75] C. S. CHANG and F. L. HINTON, "Effect of impurity particles on the finite-aspect ratio neoclassical ion thermal conductivity in a tokamak", Phys. Fluids, 29 (1986) 3314.

[76] H. TOWNER, et al., "Data analysis on TFTR using the SNAP transport code", Rev. Sci. Instrum. 63 (1992) 4753. 
[77] W. A. HOULBERG, K.-C. SHAING, S. P. HIRSHMAN, and M. C. ZARNSTORFF,

"Bootstrap current and neoclassical transport in tokamaks of arbitrary collisionality and aspect ratio", Phys. Plasmas 4 (1997) 3230.

[78] K. LACKNER, et al., "Confinement regime transitions in ASDEX”, Plasma Phys. Control. Fusion 31 (1989) 1629.

[79] K. LACKNER and N. A. O. GOTTARDI, "Tokamak Confinement in Relation to Plateau Scaling”, Nuclear Fusion 30 (1990) 767.

[80] N. KARULIN, “Transport modeling of stellarators with ASTRA”, IPP 2/328, Max-Planck Institut fur Plasmaphsyik, Garching, Germany (December 1994).

[81] E. A. LAZARUS, et al., "Simulation of a Discharge for the NCSX Stellarator", Fusion Sci. Technol. 46 (2004) 209-214.

[82] R. R. PARKER, et al., "Progress in tokamak research at MIT", Nuclear Fusion 25 (1985) 1127.

[83] T. H. STIX, "Heating of Toroidal Plasmas by Neutral Injection”, Plasma Physics, 14 (1972) 367.

[84] A. H. BOOZER and G. KUO-PETRAVIC, "Monte Carlo evaluation of transport coefficients", Phys. Fluids 24 (1981) 851.

[85] Z. LIN, W. M. TANG, and W. W. LEE, "Gyrokinetic particle simulation of neoclassical transport”, Phys. Plasmas, 2 (1995) 2975.

[86] W. LOTZ and J. NUEHRENBERG, "Monte Carlo computations of neoclassical transport", Phys. Fluids, 31 (1988) 2984.

[87] S. P. HIRSHMAN, et al., "Plasma transport coefficients for nonsymmetric toroidal confinement systems", Phys. Fluids, 29 (1986) 2951. 
[88] W. I. VAN RIJ and S. P. HIRSHMAN, "Variational bounds for transport coefficients in three-dimensional toroidal plasmas”, Phys. Fluids, B 1 (1989) 563.

[89] H. MAASSBERG, et al., "Density control problems in large stellarators with neoclassical transport", Plasma Phys. Control. Fusion 41 (1999) 1135.

[90] V. TRIBALDOS, “Monte Carlo estimation of neoclassical transport for the TJ-II

stellarator", Phys. Plasmas 8 (2001) 1229. 

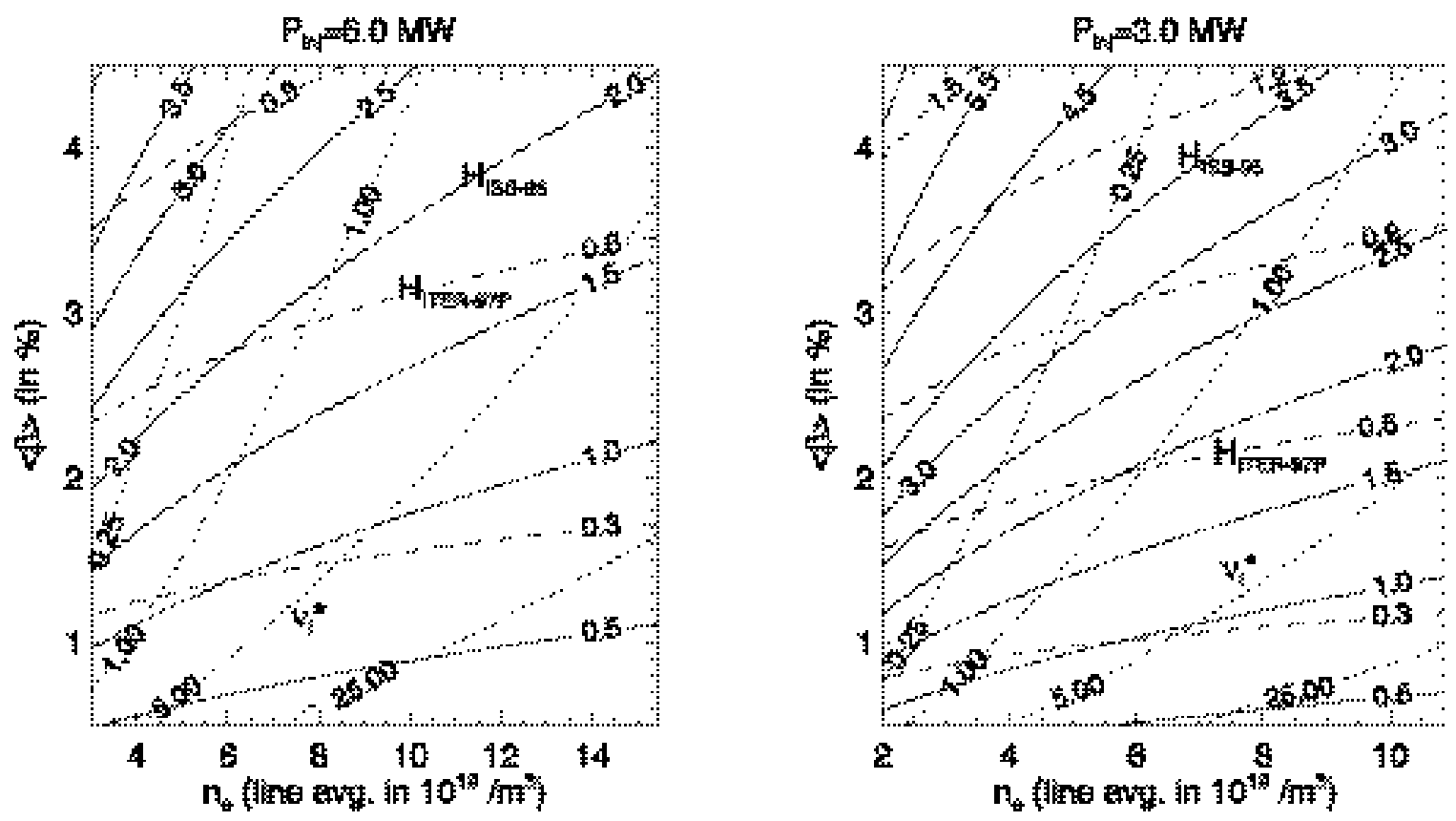

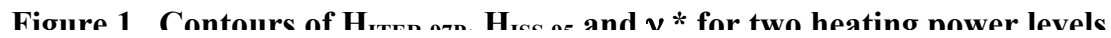




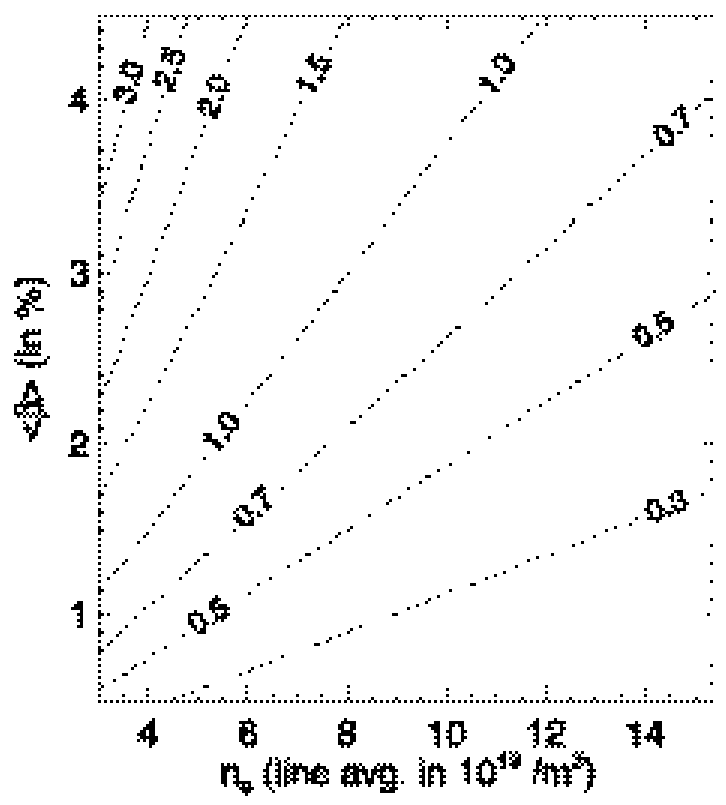

Figure 2 


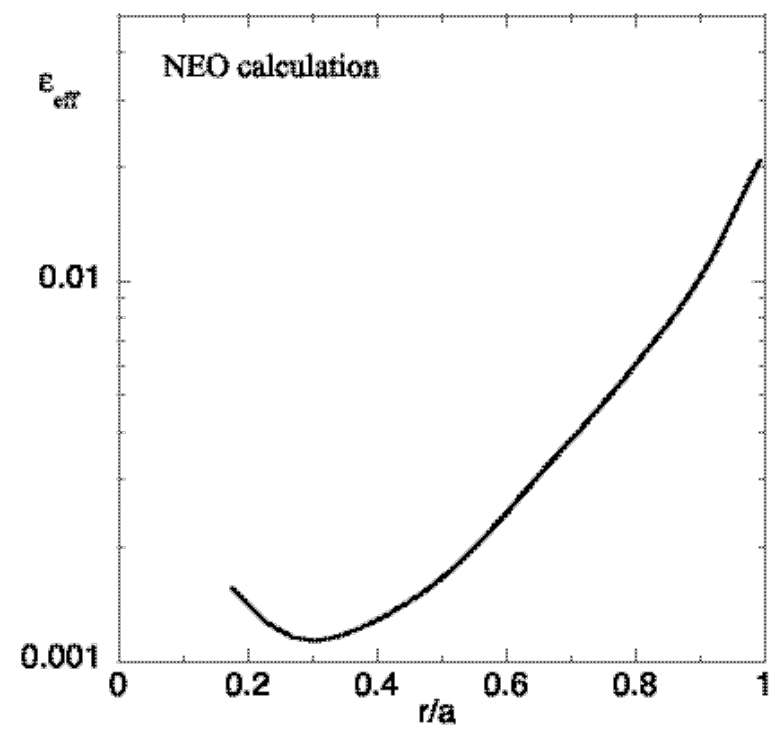

Figure 3 

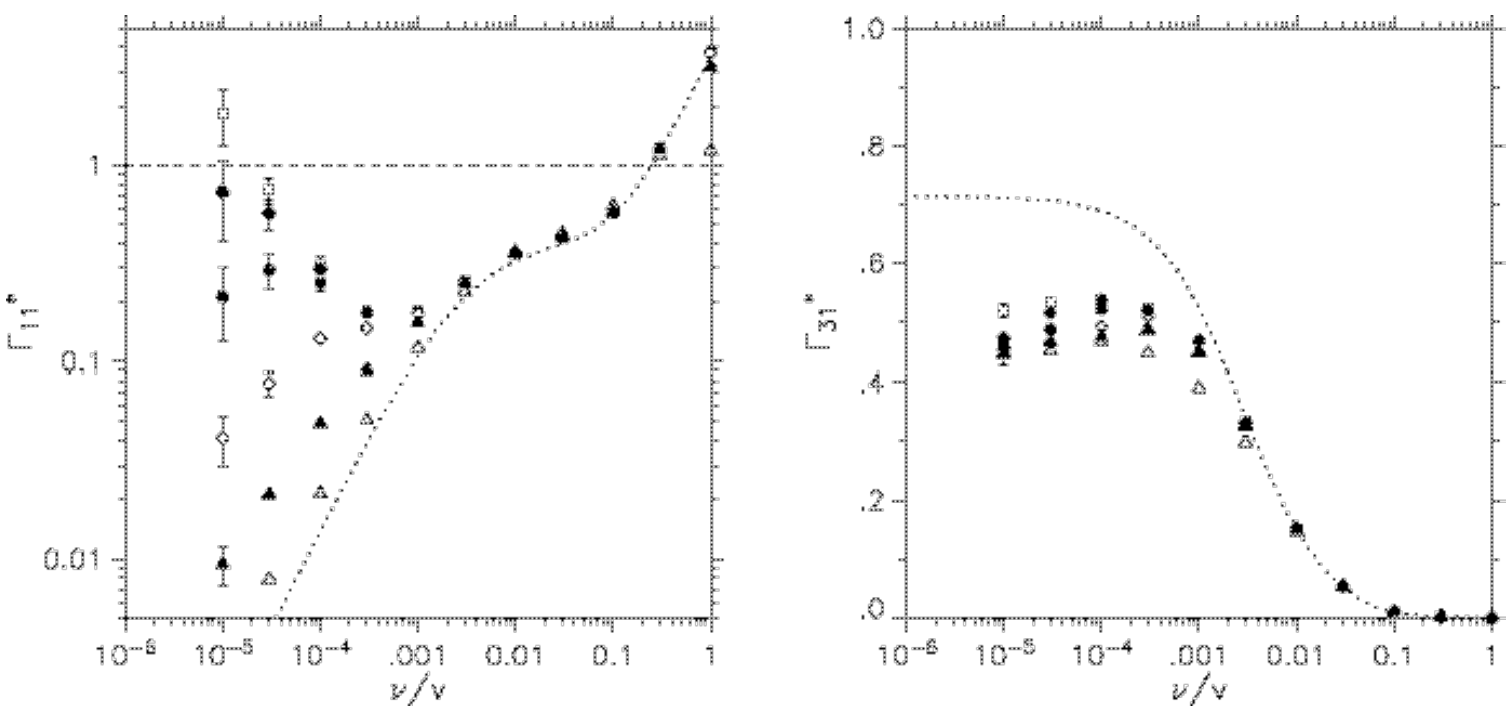

Figure 4. DKES results for NCSX at $r=a / 2$ : (a) mono-energetic particle transport coefficient normalized to the plateau value of an equivalent elongated axisymmetric configuration, and (b) the normalized bootstrap current coefficient. The abscissa is the inverse of the mean free path. Radial electric field values: $\mathrm{E}_{\mathrm{r}} /(\mathrm{Bv})=0$ red squares, $3 \times 10^{-4}$ yellow filled diamonds, $1 \times 10^{-3}$ green circles, $3 \times 10^{-3}$ dark blue diamonds, $1 \times 10^{-2}$ blue filled triangles, $3 \times 10^{-2}$ pink open triangles. 

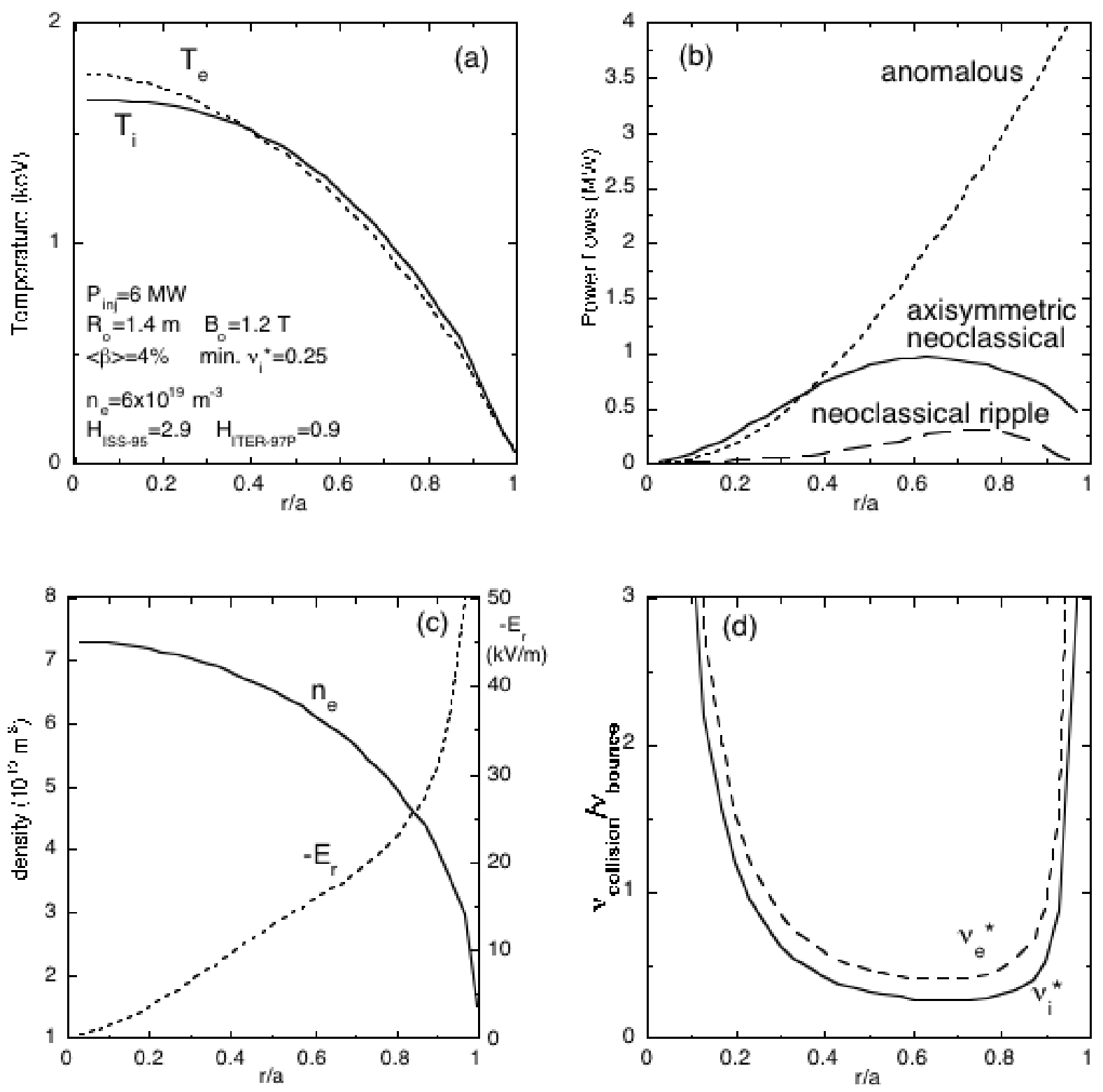

Figure 5. Profiles for standard high beta plasma. 

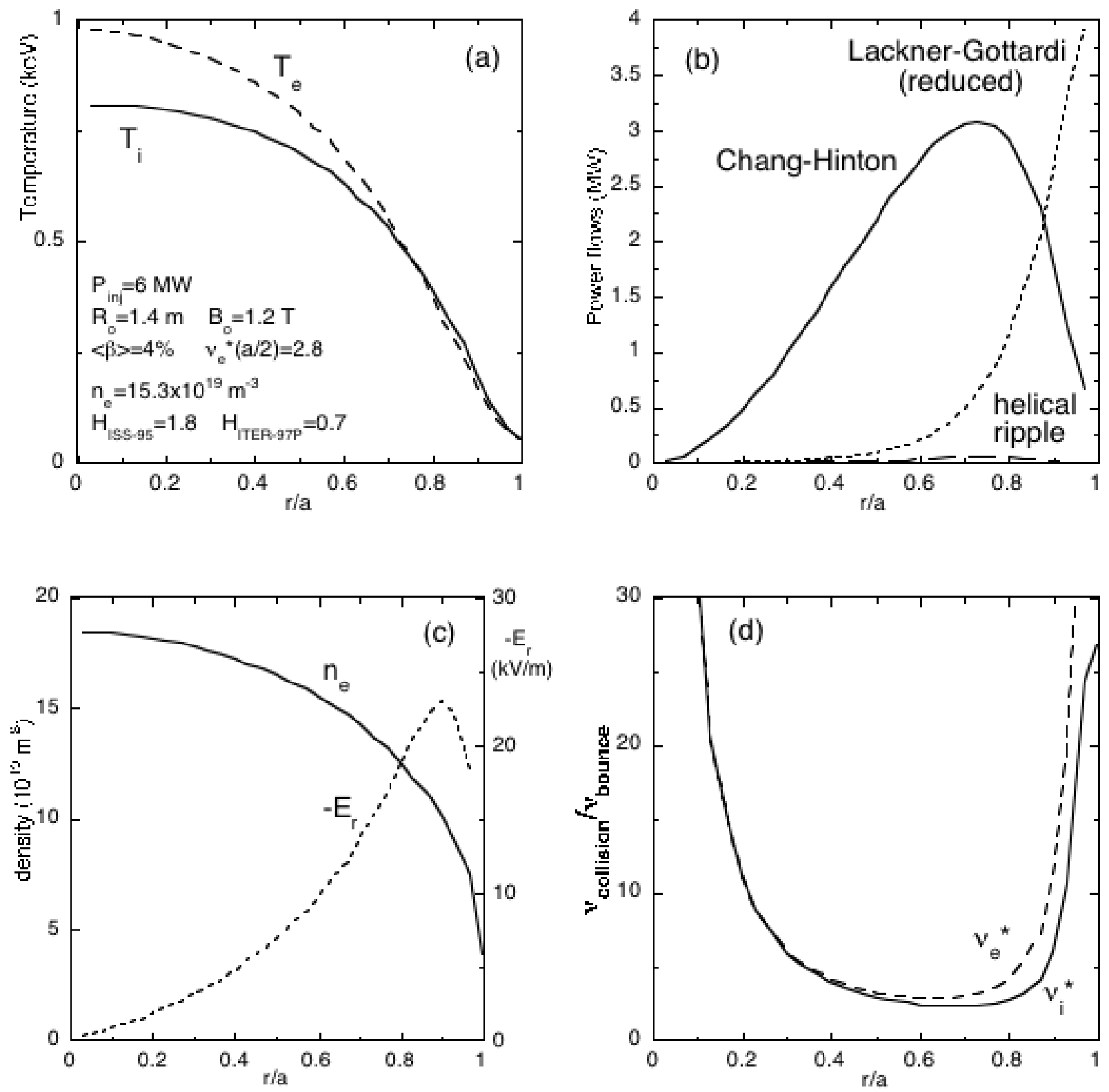

Figure 6. Profiles for high density, high beta plasma 